\title{
56 Ausdauersport und Herz
}

\author{
(c) Springer-Verlag GmbH Deutschland, ein Teil von Springer Nature 2018 \\ D. Mathias, Fit und gesund von 1 bis Hundert \\ https://doi.org/10.1007/978-3-662-56307-6_56
}

Nach der European Cardiovascular Disease Statistics 2017 sind in Europa allein 45 \% aller Todesfälle auf Herzkrankheiten zurückzuführen. Regelmäßige körperliche Aktivitäten sind deshalb wegen der vielen positiven Effekte auf das Herz eine optimale Maßnahme der Prävention. Denn der Herzmuskel kann sich je nach Trainingsleistung von $300 \mathrm{~g}$ bis auf $500 \mathrm{~g}$ vergrößern und das Herzvolumen sich von $600 \mathrm{ml}$ bis auf $1300 \mathrm{ml}$ erweitern. Die Kammerwände erfahren eine Verstärkung bis zu $20 \%$ und das Schlagvolumen erhöht sich von 60 bis auf $110 \mathrm{ml}$. Diese Ökonomisierung der Herzarbeit führt in der Summe zur Blutdrucksenkung und zur Abnahme der Herzfrequenz.

Nach Mitteilung der Europäischen Gesellschaft für Kardiologie verursachen folgende fünf Risikofaktoren drei Viertel aller schweren Herzkrankheiten:

\section{- Rauchen \\ - Diabetes \\ - Bluthochdruck \\ - Hohes LDL-Cholesterin \\ - Niedriges HDL-Cholesterin}

Die Gesellschaft empfiehlt deshalb immer wieder:

- Vermeidung von Tabakkonsum in jeglicher Form

- Abwechslungsreiche Ernährung, mit wenig gesättigten Fetten, aber mit viel Vollkornprodukten, Gemüse, Obst und Fisch

- 2,5-5 Stunden mäßig intensive körperliche Aktivitäten pro Woche

- Blutdruckwerte unter 140/90 mmHg

- Senkung zu hoher LDL-Cholesterinwerte ( Kap. 18)
Die Bedeutung dieser Empfehlungen spiegelt sich mit diesbezüglich positiven Ergebnissen in der seit 1985 laufenden Whitehall-II-Studie wider (Hardoon et al. 2012).

In Bezug auf Herz-Kreislauf-Erkrankungen profitieren die Menschen sehr vom risikosenkenden Effekt des Sports, Frauen und Männer in gleicher Weise (Hamer et al. 2014; Lin et al. 2016). Eine Metaanalyse mit den Daten von ca. 370.000 Personen über eine Zeit von 13 Jahren zeigt, dass das Risiko eines Herzversagens umso geringer ist, je mehr regelmäßig trainiert wird (Pandey et al. 2015). In einer US-amerikanischen Studie mit 11.049 Männern und einer durchschnittlichen Beobachtungszeit von 25 Jahren waren die kardiovaskulären Sterberaten der körperlich sehr fitten älteren Männer in etwa halbiert gegenüber denen der leistungsschwachen Probanden (Berry et al. 2011). Sport wirkt bei koronaren Herzkrankheiten oder bei der Rehabilitation nach Schlaganfällen genauso gut oder sogar noch besser als Medikamente (Naci und Ioannidis 2013; Möbius-Winkler et al. 2016).

Nicht Sport treibende Menschen haben häufiger einen zu schnellen Ruhepuls ( $\triangleright$ Kap. 57). Dieser erhöht u. a. die Risiken für koronare Herzerkrankungen, Herzinfarkt oder Schlaganfall, verbunden mit jeweils höheren Sterberaten (Hsia et al. 2009; Aune et al. 2017). Der Zusammenhang zwischen Herzfrequenz und Todesrate besteht auch bei jüngeren Menschen. In einer großen norwegischen Studie wurden unter diesem Aspekt 18 Jahre lang 50.088 Personen im Alter ab 20 Jahren beobachtet. Ein Ruhepuls von 101 Schlägen pro Minute steigerte hier das Sterberisiko bei Männern um 73 \% gegenüber Männern mit einer Ruhefrequenz von 61-72 Schlägen (Nauman et al. 2010). 